\title{
Fuzzy logic based on-line fault detection and classification in transmission line
}

\author{
Shuma Adhikari ${ }^{1 *} \mathbb{D}$, Nidul Sinha ${ }^{2}$ and Thingam Dorendrajit ${ }^{1}$
}

\author{
${ }^{*}$ Correspondence: \\ mom_shu@yahoo.co.in \\ ${ }^{1}$ Department of Electrical \\ Engineering, National \\ Institute of Technology \\ Manipur, Imphal, Manipur, \\ India \\ Full list of author information \\ is available at the end of the \\ article
}

\begin{abstract}
This study presents fuzzy logic based online fault detection and classification of transmission line using Programmable Automation and Control technology based National Instrument Compact Reconfigurable i/o (CRIO) devices. The LabVIEW software combined with CRIO can perform real time data acquisition of transmission line. When fault occurs in the system current waveforms are distorted due to transients and their pattern changes according to the type of fault in the system. The three phase alternating current, zero sequence and positive sequence current data generated by LabVIEW through CRIO-9067 are processed directly for relaying. The result shows that proposed technique is capable of right tripping action and classification of type of fault at high speed therefore can be employed in practical application.
\end{abstract}

Keywords: Fault, Power system protection, CRIO, Fuzzy logic

\section{Background}

Modern power system is a complex network and requires high-speed, precise, and reliable protective system. Faults in power system are unavoidable and overhead transmission line faults are generally higher compare to other major components. Due to recent technology advances, new and improved devices for protection of power system are being designed and developed. Fault classification in double circuit line with conventional techniques is difficult due to mutual coupling between the two circuits (Jain 2013). This mutual coupling is compensated by taking zero sequence current into account. The goal of system protection includes detection, classification and identification of fault with minimum time delay. In order ensure stability and continuity of service, the faulty area of the circuit should be isolated without time delay. Various methods of fault detection, classification and isolation have been reported in literature. The most commonly used techniques for fault classification are: (1) artificial neural networks (Dalstein and Kuliche 1995; Song et al. 1996; Osman et al. 2005; Mahanty and Gupta 2004; Yadav and Dash 2014; Jamil et al. 2015) (2) wavelet transform techniques (Youssef 2003; Chanda et al. 2005; Liang et al. 1998; Zhao et al. 2000; Eristi 2013; Yadav and Swetapadma 2015a; Saber et al. 2015; Koley et al. 2015) (3) fuzzy and neuro-fuzzy techniques (Nguyen and Liao 2010; Mahanty and Gupta 2006). The neural network approach for fault classification is established as a successful methodology but it requires tedious training effort, hence it is time consuming and adds to the computation complexity. Similarly wavelet

(c) 2016 The Author(s). This article is distributed under the terms of the Creative Commons Attribution 4.0 International License (http://creativecommons.org/licenses/by/4.0/), which permits unrestricted use, distribution, and reproduction in any medium, provided you give appropriate credit to the original author(s) and the source, provide a link to the Creative Commons license, and indicate if changes were made. 
transform techniques are computationally complex. The fuzzy logic based fault classification techniques are comparatively simpler as it requires only some linguistic rules. In (Ferrero et al. 1995) identified the nature of fault (whether LG or LLG), but the involved phases in the fault could not been identified and phase fault is not considered. In (Wang and Keerthipala 1998) reported the improved technique based on fuzzy-neural approach and considered both the symmetrical and unsymmetrical fault. But this method required extra effort to obtain training of ANN. In (Dash et al. 2000) showed all the ten types of fault identification by fuzzy-neural approach. In (Das and Reddy 2005; Yadav and Swetapadma 2015b; Saradarzadeh and Sanaye-Pasand 2014) proposed fuzzy logic methodology to identify the ten types of faults.

In this paper fuzzy logic based fault detection and classification on real time has been proposed. Post-fault three phase currents; zero sequence and positive sequence current samples are taken into account for fault classification. The proposed logic detects and classifies the faults at maximum delay of $100 \mathrm{~ms}$ or less with higher accuracy and also this speed can be further increased and detection time can be improved. Real time data acquisition ensures control within specified time limit.

\section{Methods}

The method adopted for the study is applied on single line diagram shown in Fig. 1. 20 numbers of different faults have been created on test-bed for tuning the fuzzy membership function and fuzzy rules. The Data are acquired through CRIO and post fault data generated for different types of fault are used to evaluate the performance of the proposed fuzzy logic based fault classification system. The power system is developed taking into consideration the acquired data as shown in Fig. 1 in Lab view software. The fuzzy logic based fault classification is first experimented i.e. on offline environment for finding the optimal system. This optimal fuzzy logic based classification system is then applied on the system for any fault on real time. It is observed during the analysis of the data that depending on the type of fault i.e. line to ground faults, line to line faults, line to line to ground faults or three phases fault, the waveform changes accordingly. It is significant to mention that during fault the voltage tends to reduce to zero and current tends to rise.

Different types of faults are characterized in terms of $\delta_{1}, \delta_{2}, \delta_{3}$ and $\delta_{4}$, which calculations are shown below (Susilo et al. 2013). Post fault current samples are solved as below.

$$
\delta_{1}=\frac{\mathrm{I}_{\mathrm{a}}-\mathrm{I}_{\mathrm{b}}}{\max \left(I_{a}, I_{b}, I_{c}\right)}
$$

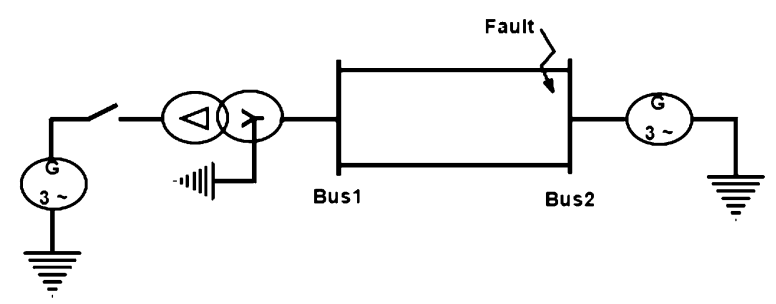

Fig. 1 Single line diagram of two bus system 


$$
\begin{aligned}
& \delta_{2}=\frac{\mathrm{I}_{\mathrm{b}}-\mathrm{I}_{\mathrm{c}}}{\max \left(I_{a}, I_{b}, I_{c}\right)} \\
& \delta_{3}=\frac{\mathrm{I}_{\mathrm{c}}-\mathrm{I}_{\mathrm{a}}}{\max \left(I_{a}, I_{b}, I_{c}\right)} \\
& \delta_{4}=\frac{\mathrm{I}_{0}}{\mathrm{I}_{1}}
\end{aligned}
$$

where $I_{a}, I_{b}$, and $I_{c}$ represent the sample of three phase currents. $I_{0}$ and $I_{1}$ are zero sequence and positive sequence current. Fuzzy rule based method for fault classification is developed on the basis of $\delta_{1}, \delta_{2}, \delta_{3}, \delta_{4}$. Zero sequence current, $\mathrm{I}_{0}$ has been taken into account to detect the presence of ground fault and $\delta_{4}$ represents the ground fault detection.

Fuzzy rule base for fault classification:

- If $\delta_{1}$ is high and $\delta_{2}$ is medium and $\delta_{3}$ is low and $\delta_{4}$ is high it is an $\mathrm{L}_{\mathrm{a}}-\mathrm{G}$ fault;

- If $\delta_{1}$ is low and $\delta_{2}$ is high and $\delta_{3}$ is medium and $\delta_{4}$ is high it is an $\mathrm{L}_{\mathrm{b}}-\mathrm{G}$ fault;

- If $\delta_{1}$ is medium and $\delta_{2}$ is low and $\delta_{3}$ is high and $\delta_{4}$ is high it is an $\mathrm{L}_{\mathrm{c}}-\mathrm{G}$ fault;

- If $\delta_{1}$ is medium and $\delta_{2}$ is high and $\delta_{3}$ is low and $\delta_{4}$ is low it is an $\mathrm{L}_{\mathrm{a}}-\mathrm{L}_{\mathrm{b}}$ fault;

- If $\delta_{1}$ is low and $\delta_{2}$ is medium and $\delta_{3}$ is high and $\delta_{4}$ is low it is an $\mathrm{L}_{\mathrm{a}}-\mathrm{L}_{\mathrm{c}}$ fault;

- If $\delta_{1}$ is high and $\delta_{2}$ is low and $\delta_{3}$ is medium and $\delta_{4}$ is low it is an $\mathrm{L}_{\mathrm{b}}-\mathrm{L}_{\mathrm{c}}$ fault;

- If $\delta_{1}$ is medium and $\delta_{2}$ is high and $\delta_{3}$ is low and $\delta_{4}$ is high it is an $\mathrm{L}_{\mathrm{a}}-\mathrm{L}_{\mathrm{b}}-\mathrm{G}$ fault;

- If $\delta_{1}$ is low and $\delta_{2}$ is medium and $\delta_{3}$ is high and $\delta_{4}$ is high it is an $\mathrm{L}_{\mathrm{a}}-\mathrm{L}_{\mathrm{c}}-\mathrm{G}$ fault;

- If $\delta_{1}$ is high and $\delta_{2}$ is low and $\delta_{3}$ is medium and $\delta_{4}$ is high it is an $\mathrm{L}_{\mathrm{b}}-\mathrm{L}_{\mathrm{c}}-\mathrm{G}$ fault;

- If $\delta_{1}$ is medium and $\delta_{2}$ is medium and $\delta_{3}$ is medium and $\delta_{4}$ is low it is an $\mathrm{L}_{\mathrm{a}}-\mathrm{L}_{\mathrm{b}}-\mathrm{L}_{\mathrm{c}}$ fault;

The triangular membership function has been used to present different fuzzy variables in the antecedent and consequent parts of the fuzzy rules as shown in Fig. 2. Mendal (1995) describes the triangular membership function as triplets with respect to the points $A, B$ and $C$. It is observed that points $A$ and $C$ have membership value of 0.0 while point B has membership value of 1.0. Extensive study has been carried out to select proper triplets values of triangular membership function of $\delta_{1}, \delta_{2}, \delta_{3}$ and $\delta_{4}$. The selected triplets for triangular membership function of fuzzy variables in antecedents parts and consequent part are shown in Tables 1, 2 and 3 respectively.

\section{Block diagram of fuzzy logic based fault classification}

Figure 3 presents the block diagram of the proposed methodology. The ADC (analog to digital converter) is connected with FPGA (field-programmable gate array) hardware. The FPGA can directly access the ADC acquired values and send it to RT (real 


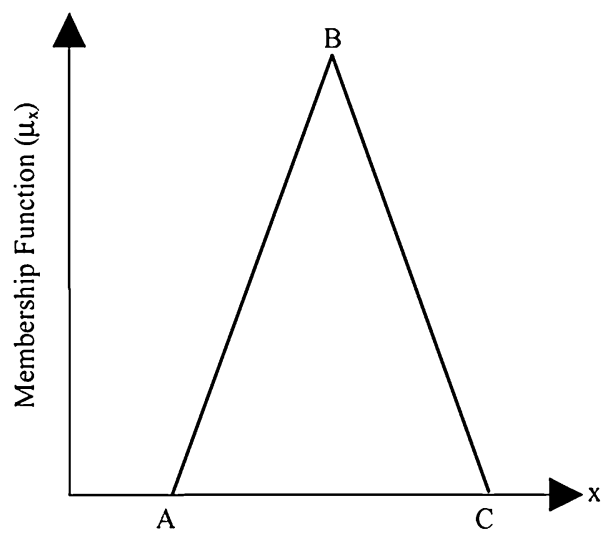

Fig. 2 Triangular fuzzy membership function

Table 1 Fuzzy variables in the antecedent parts of fuzzy rules for $\delta_{1}, \delta_{2}, \delta_{3}$

\begin{tabular}{lllr}
\hline Input variables & \multicolumn{2}{l}{ Triangular triplets } & C \\
\cline { 2 - 4 } & A & B & -0.56 \\
\hline Low & -1 & 1 & 0.61 \\
Medium & -0.61 & 1 & 1 \\
High & 0.54 & 1 & 1
\end{tabular}

Table 2 Fuzzy variables in the antecedent parts of fuzzy rules for $\boldsymbol{\delta}_{\mathbf{4}}$

\begin{tabular}{llll}
\hline Input variables & \multicolumn{2}{l}{ Triangular triplets } & C \\
\cline { 2 - 4 } & A & B & 0.1 \\
\hline Low & -2 & 1 & 3 \\
High & -0.01 & 1 & \\
\hline
\end{tabular}

Table 3 Fuzzy variables in the consequent parts of fuzzy rules

\begin{tabular}{lccr}
\hline Fuzzy variables & Triplets & & \\
\cline { 2 - 4 } & A & B & C \\
\hline$L_{a}-G$ & 6.5 & 7 & 7.5 \\
$L_{b}-G$ & 4.5 & 5 & 5.5 \\
$L_{c}-G$ & 2.5 & 3 & 3.5 \\
$L_{a}-L_{b}$ & 63.5 & 64 & 64.5 \\
$L_{a}-L_{c}$ & 60.5 & 61 & 61.5 \\
$L_{b}-L_{c}$ & 65.5 & 66 & 66.5 \\
$L_{a}-L_{b}-G$ & 76.5 & 77 & 77.5 \\
$L_{a}-L_{c}-G$ & 94.5 & 95 & 95.5 \\
$L_{b}-L_{c}-G$ & 96.5 & 97 & 97.5 \\
$L_{a}-L_{b}-L_{c}$ & 73.5 & 74 & 74.5 \\
\hline
\end{tabular}




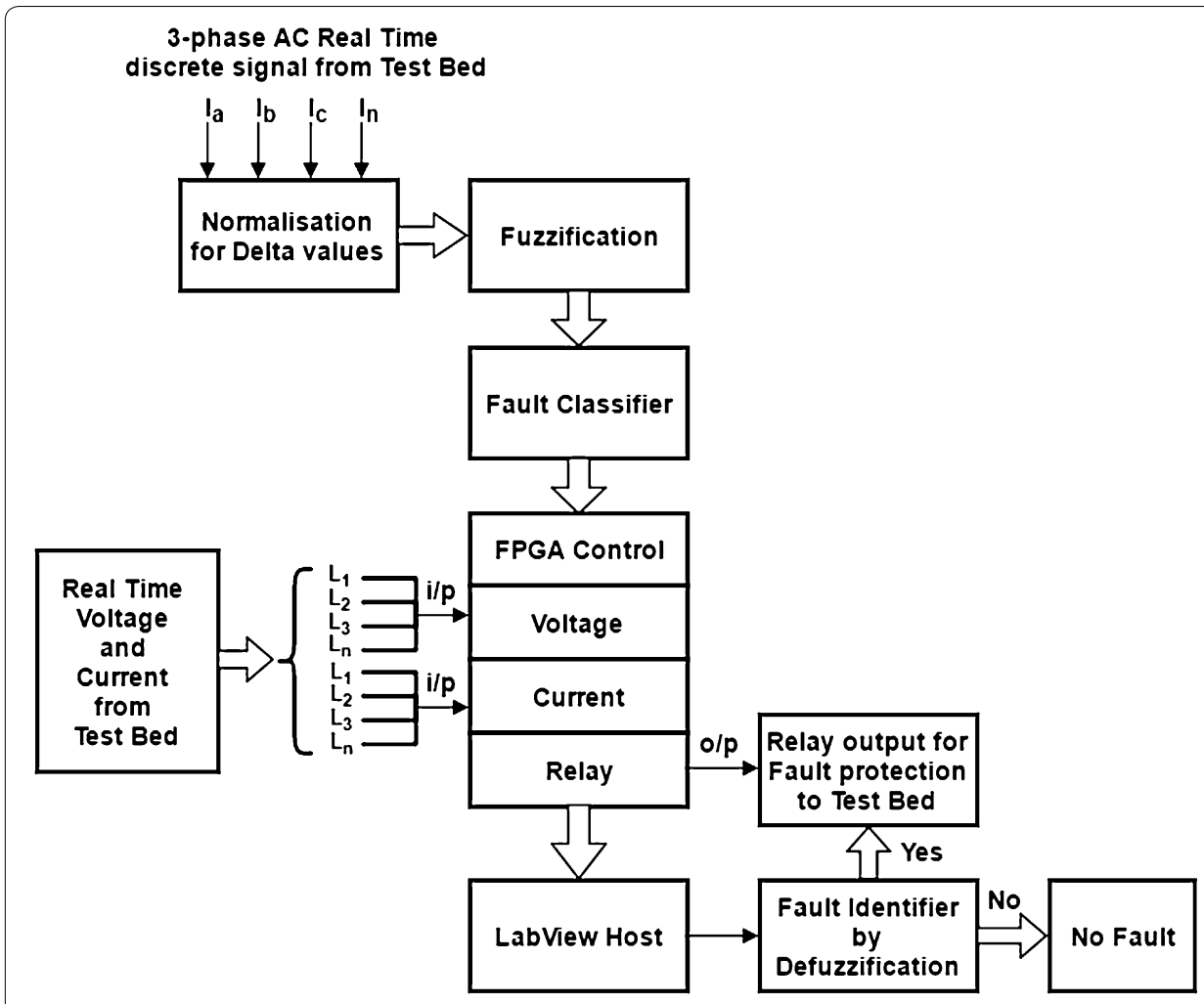

Fig. 3 Block diagram of the proposed methodology

time) processor. The FPGA uses a $40 \mathrm{M} \mathrm{Hz}$ clock for its operation. The Sampling rate of FPGA is set at $10 \mathrm{kS} / \mathrm{s}$ which give 200 samples for each cycle. For every sample the FPGA acquire value is put into a FIFO (fast in fast out) queue, which can be accessed from the RT processor. The RT processor polls 1000 values for each channel, which means 5 consecutive cycles of $50 \mathrm{~Hz}$ signal. Once 5 cycle data is present, the RT processes the data and measures the RMS value of the signal acquired, and also checks for fault conditions. The sampling rate and the number of sample to detect the fault can be varied using the user control; this gives an option to change the parameters of the fault detector leading to improvement of efficiency, accuracy and response time. Three phase current data from test-bed have been acquired through CRIO. The signals acquired are normalized and different faults are characterized in terms of $\delta_{1}, \delta_{2}, \delta_{3}$ and $\delta_{4}$. After analysis of data obtained, triplet values are selected in antecedent and consequent parts to represent various fuzzy variables. Rules base are then prepared for classifying the fault type. After successful compilation of simulation fuzzy logic, the generated logic is dumped to the Field Programmable Gate array (FPGA) control LabVIEW hardware. In CRIO three modules have been used for high voltage data acquisition modules, high current data acquisition module and relay switch module for protection. The real time, line voltage and current values from the test bed are used as input to the FPGA control. When fault 
occurs in the system, relay switch module detect fault and the relay will trip after intentional $5 \mathrm{~ms}$ delay. The type of fault occurring in the system will be displayed on the host PC.

\section{Real time monitoring and controlling}

Laboratory Virtual Instrument Engineering Workbench (LabVIEW) is a powerful and flexible instrumentation and analysis software application tool which was developed in 1986 by the National Instruments. LabVIEW is extremely flexible and commonly used for data acquisition, instrument control, data processing and industrial automation. The CRIO device can interface between computers and Test-bed set up. Figure 4 shows schematic of real time monitoring and controlling. The LabVIEW 14.01 and CRIO 9067 systems have been implemented as SCADA to monitor real time parameters of test-bed. CRIO 9067 combines a dual-core processor, a reconfigurable FPGA, and eight slots for C Series I/O modules within one chassis. The CRIO systems consist of a real time processor running Real Time Operating System (RTOS) and also a FPGA backplane for accessing the I/O s. The RTOS and FPGA work mutually to ensure real time performance. C series I/O modules used for the study are-NI 9244, NI 9227, NI 9472, NI 9482, NI 9871, NI 9467. The NI 9244 module has 3 single-ended channels, $50 \mathrm{kS} / \mathrm{s}$ per channel simultaneous sample rate with $400 \mathrm{Vrms} \mathrm{L-N,} 800 \mathrm{Vrms}$ L-L measurement range \& 24-bit resolution and NI $9227 \mathrm{C}$ Series current input module was designed to measure $5 \mathrm{~A} \mathrm{rms}$ nominal and up to 14 A peak on each channel with channel-to-channel isolation with 50 kS/s/ch simultaneous sample rate. The NI 9871 has 4-Port, RS485/RS422 Serial Interface Module and used for RS485 data monitor on MODBUS. NI 9871 has baud rates up to $3.684 \mathrm{Mbaud}$ per port. We can pass up to $1.28 \mathrm{Mbit} / \mathrm{s}$ of data between the module and CompactRIO. This ensure that the data monitored by the current and voltage monitor modules are correct and matching with the other measuring systems output. The NI 9467 provides GPS location information for measuring phasor values precisely. The digital output module and relay control module is used for protection of the grid. NI 9482 relay module is used. NI 9482 is a 4-channel, single-pole single throw (SPST) sourcing digital output module for NI Compact DAQ and CompactRIO. Each channel provides an SPST relay for switching signals up to 30 VDC (1.5 A), 60 VDC (1 A), 250 VAC (1.5 A) when all channels are being used. Figure 5 presents front panel of LabVIEW graphical user interface (GUI) created. Figure 6 shows real time program for fuzzy logic based fault classification.

\section{Hardware implementation}

We have used National Instruments Controller with $667 \mathrm{MHz}$ Dual-Core ARM CortexA9 processor running in the NI Linux Real-Time, also integrated Chassis has Artix-7

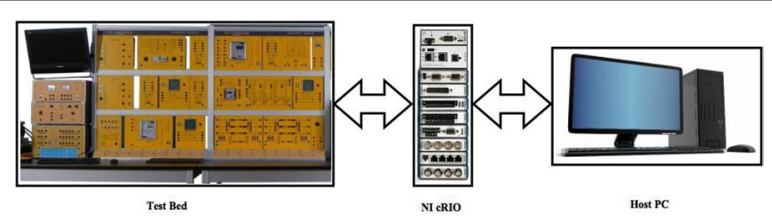

Fig. 4 Programmable automation and control technology of test-bed 


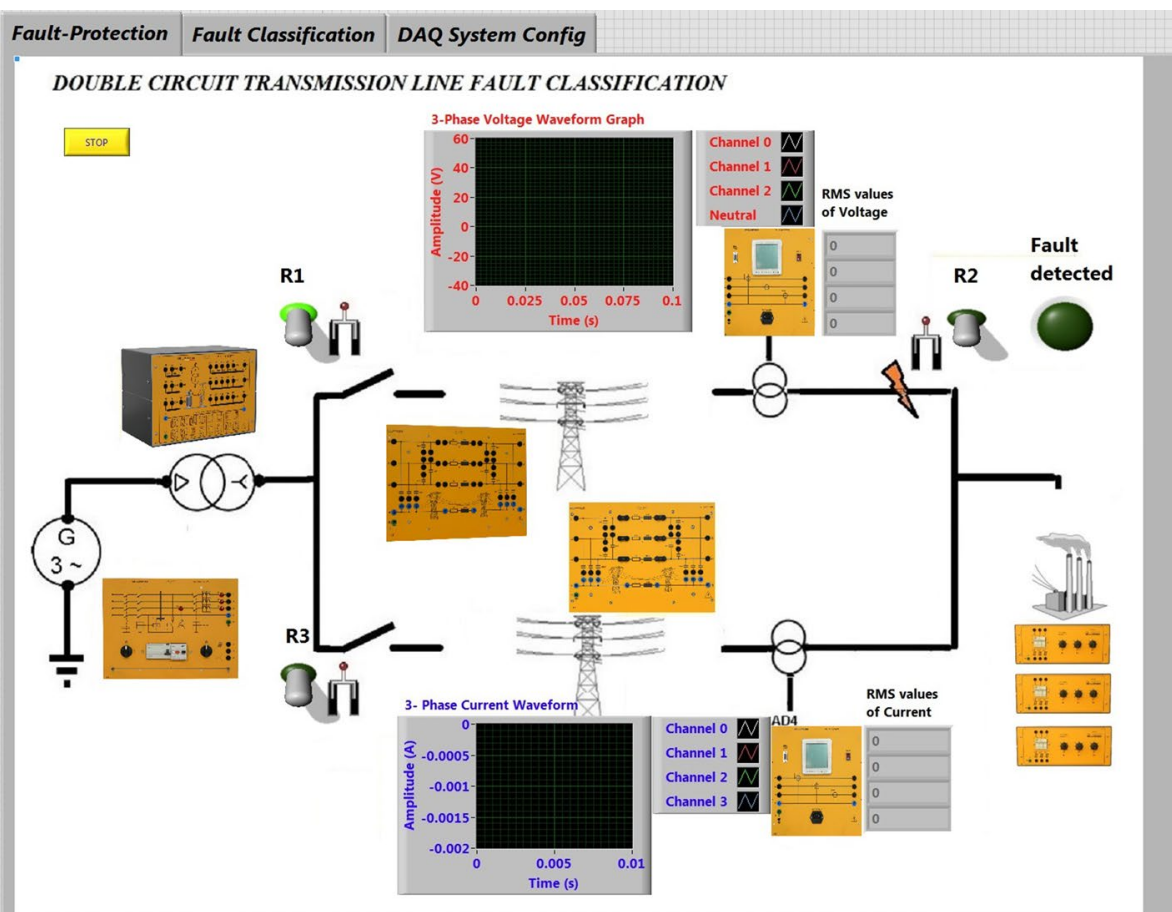

Fig. 5 Front panel of the LabvlEW GUI

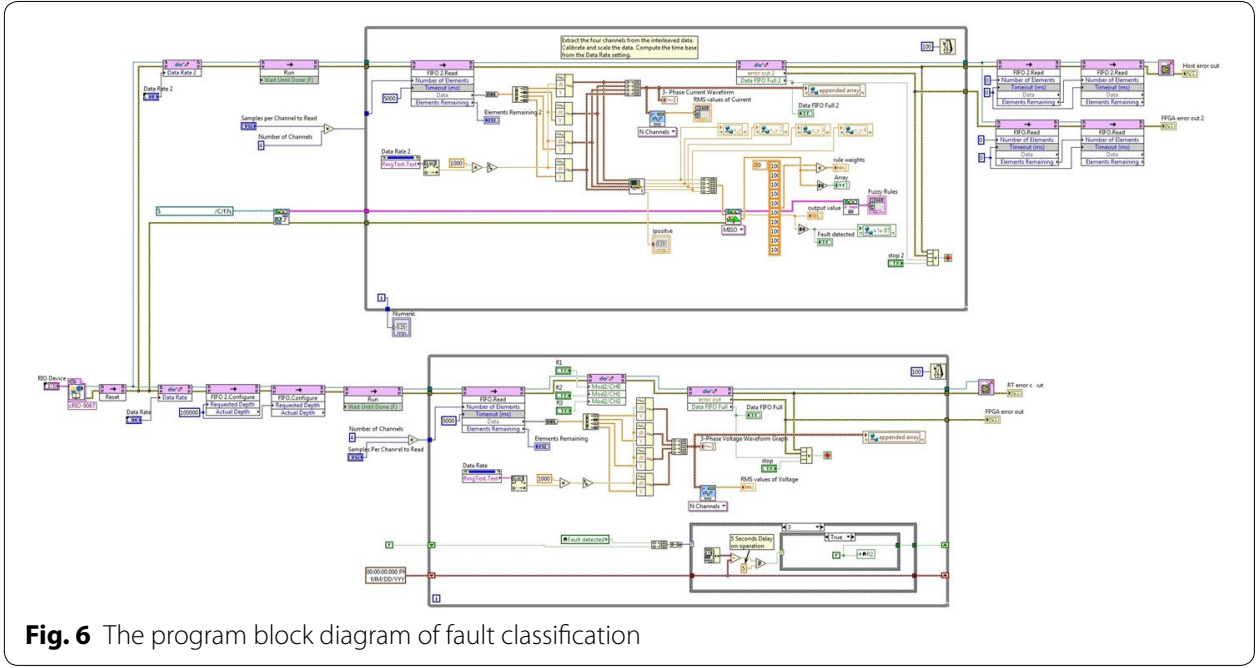

FPGA. LabVIEW 14 Version has been used for programming and implementation of logics. For the compilation process LabVIEW uses Xilinx Vivado 2013.4 as Compilation Tool. Table 4 shows the compilation result. 
Table 4 Logic utilization table

\begin{tabular}{llrrl}
\hline Sl. no & Device utilization & Used & Available & Percentage of utilization \\
\hline 1 & Total slices & 4371 & 13,300 & 32.8 \\
2 & Slice register & 11,038 & 106,400 & 10.4 \\
3 & Slice LUT's & 11,319 & 53,200 & 21.3 \\
4 & Block RAMs & 24 & 140 & 17.1 \\
5 & DSP48s & 2 & 220 & 0.9 \\
\hline
\end{tabular}

The proposed logic for fault detection and classification has been tested on an experimental transmission line module on $360 \mathrm{~km}$ of $\pi$-model. The picture of the hardware set-up is shown in the Fig. 7. Table 5 represent the data of the test-bed.

\section{Results and discussion}

The hardware set up is connected properly. Ten different faults are created as shown in Fig. 1 on $360 \mathrm{~km}$ double circuit line. For fault detection and classification LabVIEW fuzzy logic tool kit has been used and the system is protected at $5 \mathrm{~ms}$ delay triggered using LabVIEW through CRIO controller relay module. It is worth to mention that proposed logic is able to detect fault, trip the line as well as classify type of the fault occurred. Figure 8 shows the voltage waveform before introduction of fault in the system.

The voltage and current waveforms for La - G fault are presented in Figs. 9 and 10. It is observed that when fault occurs in the system, voltage of $L_{a}$ reduces and current increases. Figure 11 presents graphical result of fault classification which follow fuzzy rule base of $\mathrm{L}_{\mathrm{a}}-\mathrm{G}$ fault i.e. $\delta_{1}$ is high and $\delta_{2}$ is medium and $\delta_{3}$ is low and $\delta_{4}$ is high.

Figures 12 and 13 show voltage and current waveforms for $L_{a}-L_{b}-L_{c}$ fault. It can be seen that when fault occurs in the system, voltage of $\mathrm{L}_{\mathrm{a}}, \mathrm{L}_{\mathrm{b}}$ and $\mathrm{L}_{\mathrm{c}}$ reduces and current increases. It is also observed in Fig. 14 that $\delta 1, \delta_{2}, \delta_{3}$ and $\delta_{4}$ satisfy fuzzy rule base for $\mathrm{L}_{\mathrm{a}}-\mathrm{L}_{\mathrm{b}}-\mathrm{L}_{\mathrm{c}}$ fault.

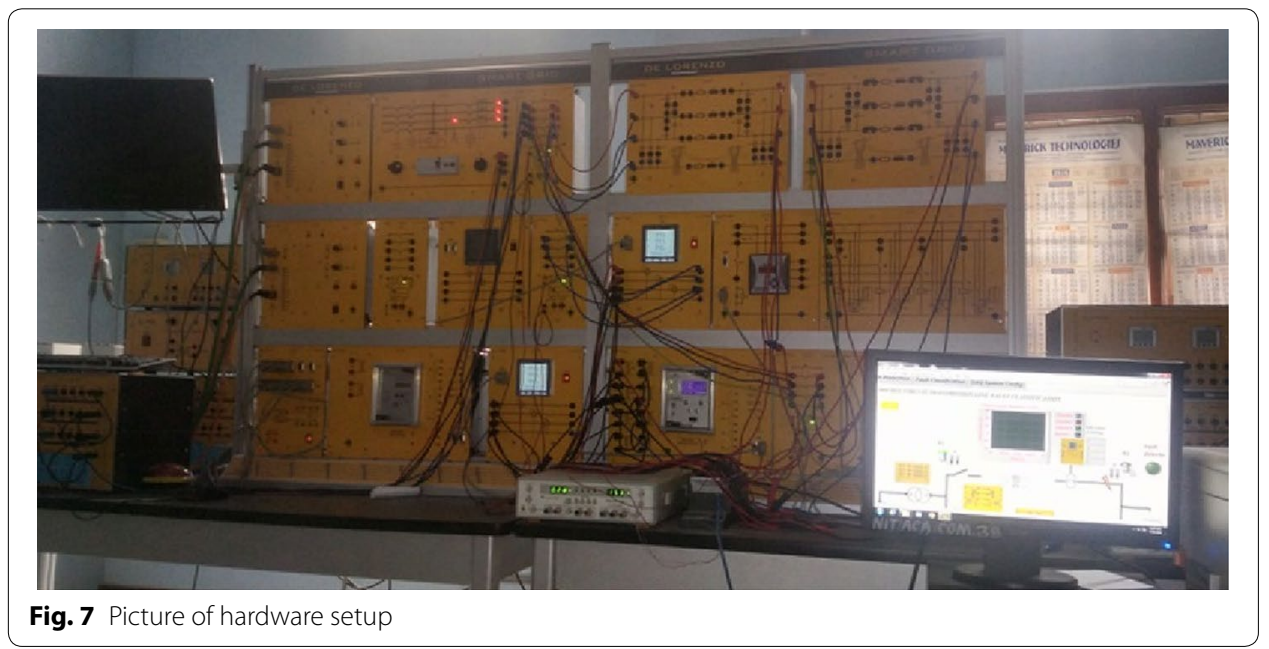


Table 5 Data of the test-bed

\begin{tabular}{ll}
\hline Scale factor & \\
Voltage & $380 \mathrm{~V}: 380 \mathrm{kV}=1: 1000$ \\
Current & $1 \mathrm{~A}: 1000 \mathrm{~A}=1: 1000$ \\
Line parameters & \\
Resistance & $\mathrm{R}_{\mathrm{L}}=13 \Omega$ \\
Inductance & $\mathrm{L}_{\mathrm{L}}=290 \mathrm{mH}$ \\
Mutual capacitance & $\mathrm{C}_{\mathrm{L}}=0.5 \mu \mathrm{F}$ \\
Earth capacitance & $\mathrm{C}_{\mathrm{E}}=1 \mu \mathrm{F}$ \\
Earth return parameter & \\
Resistance & $\mathrm{R}_{\mathrm{E}}=11 \Omega$ \\
Inductance & $\mathrm{L}_{\mathrm{E}}=250 \mathrm{mH}$ \\
Natural load & \\
$600 \mathrm{MW}$ & \\
Characteristic impedance & \\
$340 \Omega$ & \\
\hline
\end{tabular}

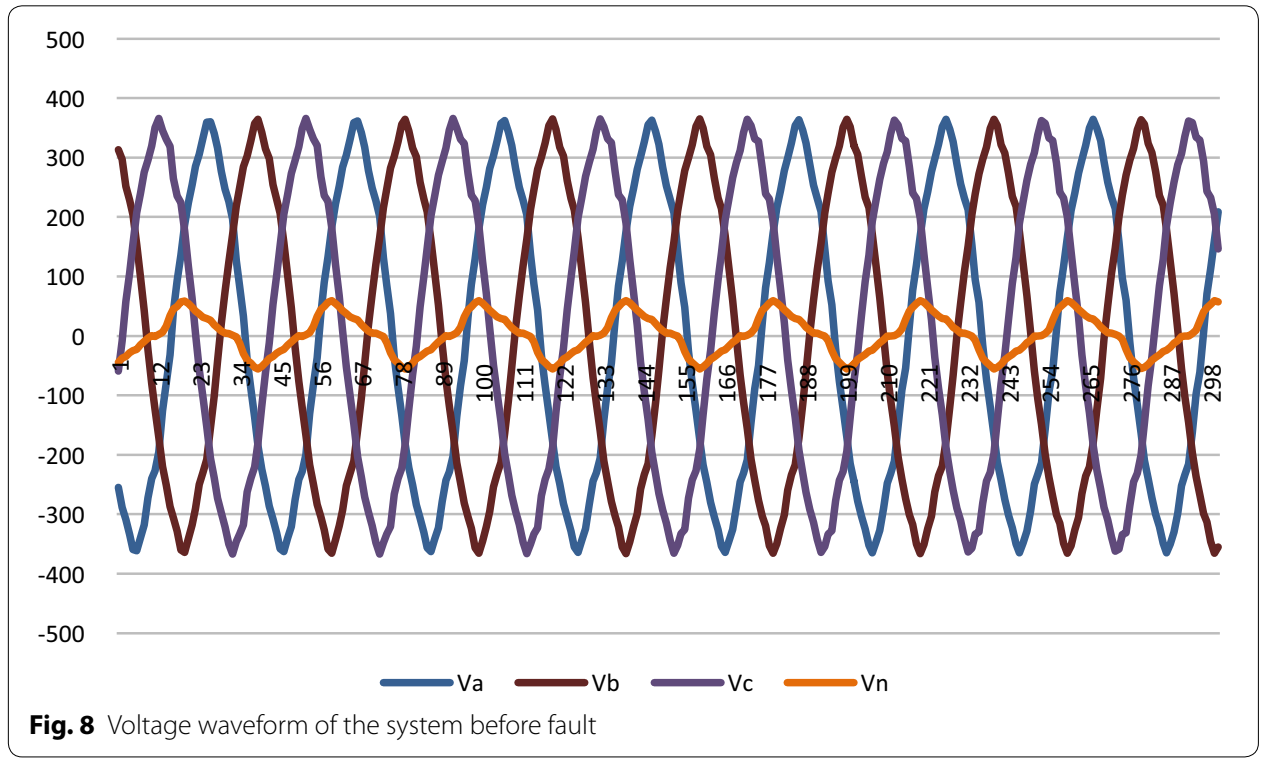

The fuzzy logic based fault identification and classification is easy and simple since it only require computation of some ratios and differences of ratios of post fault current samples. When different fault are introduced in the system the corresponding fuzzy logic output are presented in Table 6 . All the faults are checked graphically to 

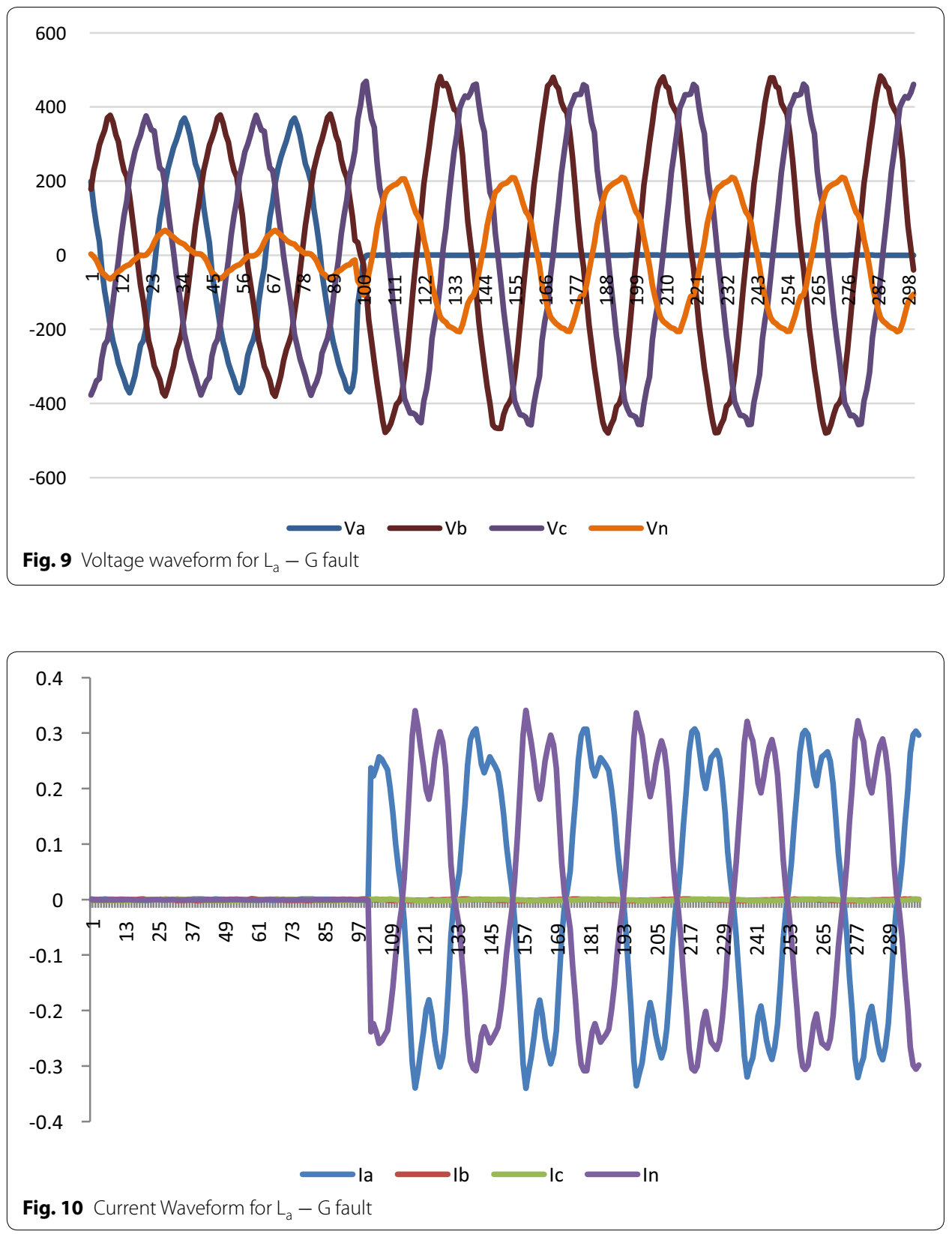

confirm the accuracy of the proposed scheme. The proposed logic detects and classifies the fault accurately. The results show that the proposed logic is efficient and appropriate. 


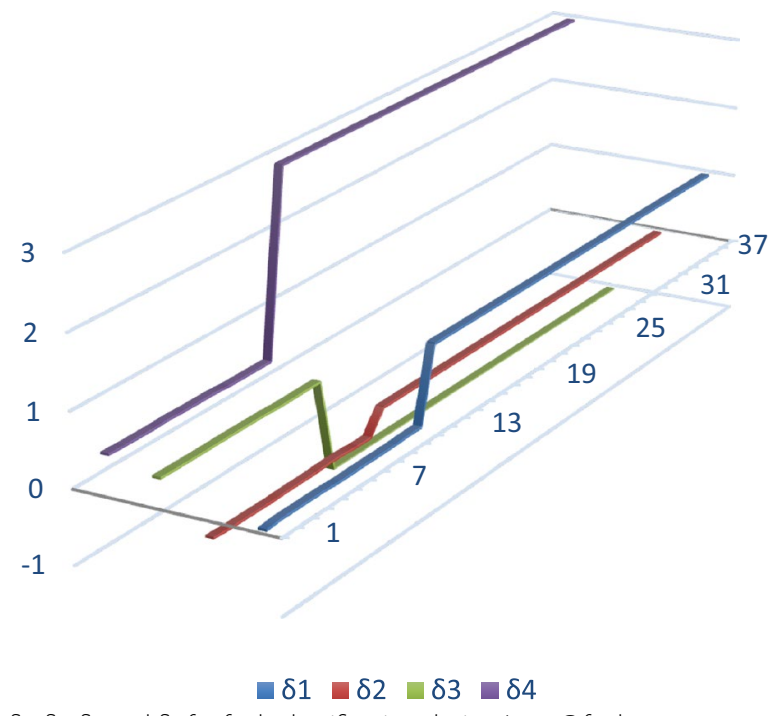

Fig. 11 Output for $\delta_{1}, \delta_{2}, \delta_{3}$ and $\delta_{4}$ for fault classification during $L_{a}-G$ fault

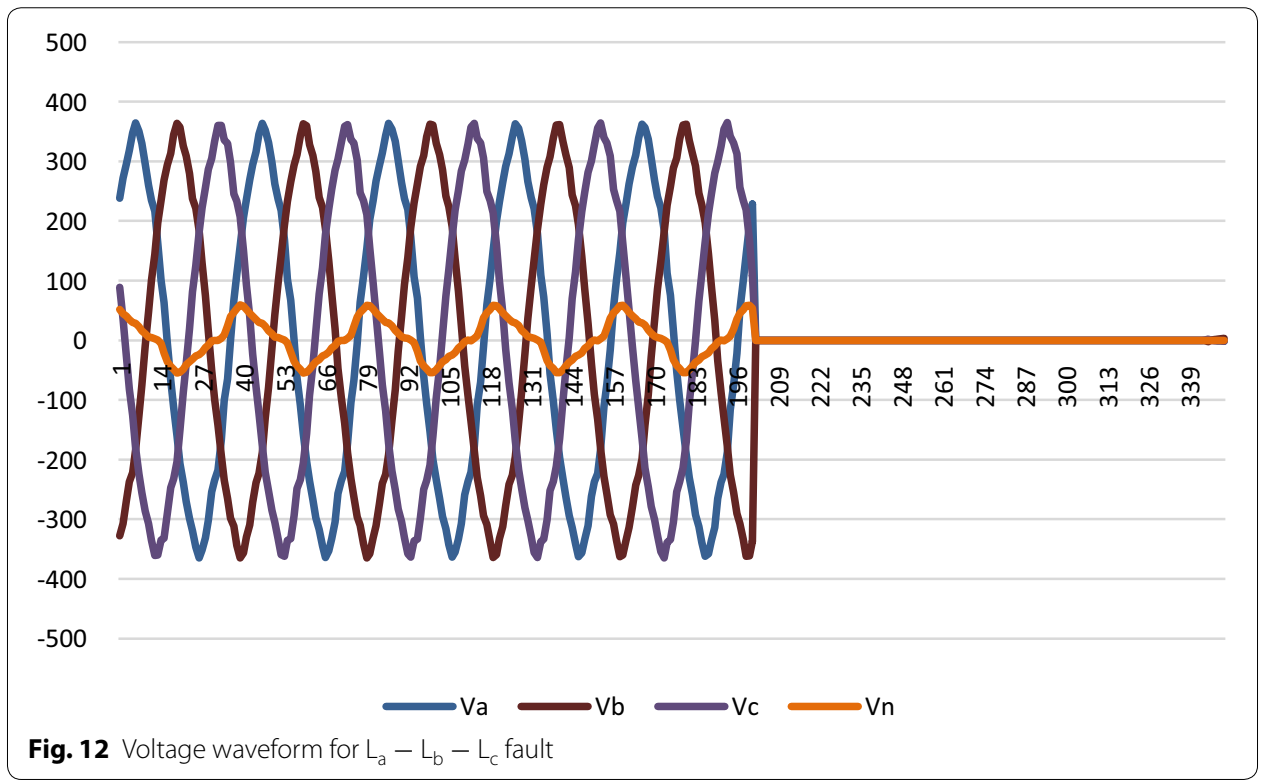

\section{Conclusion}

An approach for fault detection and classification for transmission line based on fuzzy logic is found to be very efficient and effective under different fault conditions. This technique can determine not only fault detection and fault classification but can also 

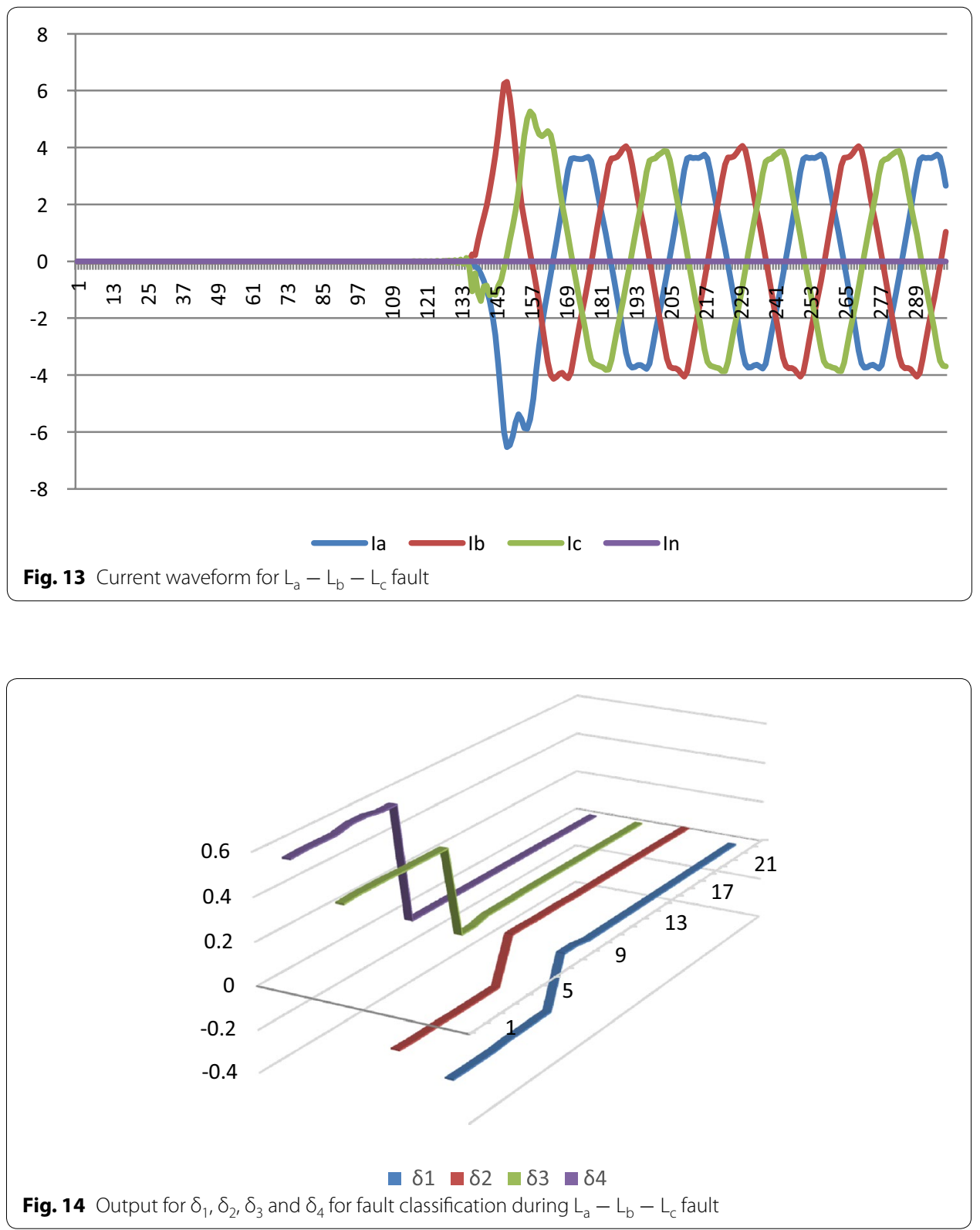

give automatic protection in real time. The system operation is fast, reliable, and secure. Proposed logic is simple since it requires only some linguistic rules. The results show that proposed techniques is simple, fast, reliable and secure. 
Table 6 Result of fuzzy logic based fault classification

\begin{tabular}{lllc}
\hline Fault type & FLS input $\left(\boldsymbol{\delta}_{\mathbf{1}}, \boldsymbol{\delta}_{\mathbf{2}}, \boldsymbol{\delta}_{\mathbf{3}}, \boldsymbol{\delta}_{\mathbf{4}}\right)$ & Fault currents $(\mathbf{l a}, \mathbf{I b}, \mathbf{I c}, \mathbf{I n})$ & FLS output \\
\hline$L_{a}-G$ & $0.98737,-0.00296,-0.98441,2.92775$ & $-0.13610,-0.00158,-0.00073,0.13671$ & 6.85 \\
$L_{b}-G$ & $-0.99090,0.98533,0.00556,2.94215$ & $0.00109,0.27380,-0.00024,-0.27600$ & 5.15 \\
$L_{c}-G$ & $-0.00264,-0.98845,0.99110,2.95408$ & $0.00012,-0.00036,-0.06103,0.03784$ & 3.30 \\
$L_{a}-L_{b}$ & $0.00017,0.99949,-0.99951,0.00034$ & $3.05688,-3.05761,-0.00134,-0.00048$ & 64.10 \\
$L_{a}-L_{c}$ & $0.99939,-0.99955,0.00015,0.00036$ & $3.19409,-0.00024,-2.77185,-0.00073$ & 66.15 \\
$L_{b}-L_{c}$ & $-0.99968,0.00012,0.99967,0.00034$ & $0.00024,-2.58276,2.58154,-0.00036$ & 61.30 \\
$L_{a}-L_{b}-G$ & $0.01731,0.98115,-0.99847,0.07258$ & $1.11511,-1.23242,-0.00231,0.11669$ & 77.10 \\
$L_{a}-L_{c}-G$ & $0.98100,-0.99869,0.01769,0.09245$ & $3.63757,-0.00085,-3.66918,0.02856$ & 95.40 \\
$L_{b}-L_{c}-G$ & $-0.99946,0.01475,0.98471,0.11095$ & $0.00158,1.94299,-1.63598,-0.31176$ & 97.10 \\
$L_{a}-L_{b}-L_{c}$ & $0.00144,0.00508,-0.00653,0.00058$ & $3.68237,-1.95690,-1.72717,-0.00036$ & 74.25 \\
\hline
\end{tabular}

\section{Authors' contributions}

SA and TD conducted the research work on the laboratory and data presented is the actual data that was obtained during the experiment. The article was then written by SA and was reviewed by NS. All authors read and approved the final manuscript.

\section{Author details}

${ }^{1}$ Department of Electrical Engineering, National Institute of Technology Manipur, Imphal, Manipur, India. ${ }^{2}$ Department of Electrical Engineering, National Institute of Technology Silchar, Silchar, Assam, India.

\section{Competing interests}

The authors declare that they have no competing interests.

Received: 23 April 2016 Accepted: 25 June 2016

Published online: 07 July 2016

\section{References}

Chanda D, Kishore NK, Sinha AK (2005) Application of wavelet multiresolution analysis for identification and classification of faults on transmission lines. Electr Power Syst Res 73:323-333

Dalstein T, Kuliche B (1995) Neural network approach to fault classification for high speed protective relaying. IEEE Trans Power Deliv 10(2):1002-1011

Das B, Reddy JV (2005) Fuzzy-logic-based fault classification scheme for digital distance protection. IEEE Trans Power Deliv 20(2):609-616

Dash PK, Pradhan AK, Panda G (2000) A novel fuzzy neural network based distance relaying scheme. IEEE Trans Power Deliv 15(3):902-907

Eristi H (2013) Fault diagnosis system for series compensated transmission line based on wavelet transform and adaptive neuro-fuzzy inference system. Measurement 46(1):393-401

Ferrero A, Sangiovanni S, Zapitelli E (1995) A fuzzy set approach to fault type identification in digital relaying. IEEE Trans Power Deliv 10(1):169-175

Jain A (2013) Artificial neural network-based fault distance locator for double-circuit transmission lines. Adv Artif Intell 2013:1-12

Jamil M, Sharma SK, Singh R (2015) Fault detection and classification in electrical power transmission system using artificial neural network. Springerplus 4(334):1-13

Koley E, Verma K, Ghosh S (2015) An improved fault detection classification and location scheme based on wavelet transform and artificial neural network for six phase transmission line using single end data only. Springerplus 4(551):1-22

Liang J, Elangovan S, Devotta JBX (1998) A wavelet multiresolution analysis approach to fault detection and classification in transmission lines. Int J Electr Power Energy Syst 20(5):327-332

Mahanty RN, Gupta PBD (2004) Application of RBF neural network to fault classification and location in transmission lines. IEEE Proc Gener Transm Distrib 151(2):201-212

Mahanty RN, Gupta PBD (2006) A fuzzy logic based fault classification approach using current samples only. Electr Power Syst Res 77(2007):501-507

Mendal JM (1995) Fuzzy logic systems for engineering: a tutorial. Proc IEEE 83(3):345-377

Nguyen T, Liao Y (2010) Transmission line fault type classification based on novel features and neuro-fuzzy system. Electr Power Compon Syst 38(6):695-709

Osman AH, Abdelazim T, Malik OP (2005) Transmission line distance relaying using on line trained neural networks. IEEE Trans Power Deliv 20(2):1257-1264

Saber A, Emam A, Amer R (2015) Discrete wavelet transform and support vector machine-based parallel transmission line faults classification. IEE J Trans Electr Electron Eng 11(1):43-48 
Saradarzadeh M, Sanaye-Pasand M (2014) An accurate fuzzy logic-based fault classification algorithm using voltage and current phase sequence components. Int Trans Electr Energy Syst 25(10):2275-2288

Song YH, Johns AT, Xuan QY (1996) Artificial neural-network-based protection scheme for controllable series-compensated EHV transmission lines. IEEE Proc Gener Transm Distrib 143(6):535-540

Susilo L, Gu JC, Huang SK (2013) Fault current characterization based on fuzzy algorithm for DOCR application. Energy Power Eng 5:932-936

Wang H, Keerthipala WWL (1998) Fuzzy neuro approach to fault classification for transmission line protection. IEEE Trans Power Deliv 13(14):1093-1104

Yadav A, Dash Y (2014) An overview of transmission line protection by artificial neural network: fault detection, fault classification, fault location, and fault direction discrimination. Adv Artif Neural Syst 2014:1-21

Yadav A, Swetapadma A (2015a) Enhancing the performance of transmission line directional relaying, fault classification and fault location schemes using fuzzy inference system. IET Gener Transm Distrib 9(6):580-591

Yadav A, Swetapadma A (2015b) A novel transmission line relaying scheme for fault detection and classification using wavelet transform and linear discriminant analysis. Ain Shams Eng J 6(1):199-209

Youssef OAS (2003) A modified wavelet-based fault classification technique. Electr Power Syst Res 64(2):165-172

Zhao W, Song YH, Min Y (2000) Wavelet analysis based scheme for fault detection and classification in underground power cable systems. Electr Power Syst Res 53(1):23-30

\section{Submit your manuscript to a SpringerOpen ${ }^{\circ}$ journal and benefit from:}

- Convenient online submission

\section{- Rigorous peer review}

- Immediate publication on acceptance

- Open access: articles freely available online

- High visibility within the field

- Retaining the copyright to your article

Submit your next manuscript at $\boldsymbol{\nabla}$ springeropen.com 